\title{
Positive Germline Selection in Pedigrees With Multiple Endocrine Neoplasia Type 2 Carrying V804M Mutation in the RET Gene
}

\author{
Leema Reddy Peddareddygari ${ }^{\mathrm{a}}$, Angela Musial Fay ${ }^{\mathrm{b}}$, Alexander L. Shifrin ${ }^{\mathrm{b}}$, \\ Raji P. Grewal ${ }^{c, d}$
}

\begin{abstract}
Background: Multiple endocrine neoplasia (MEN) type 2 is an autosomal dominant cancer syndrome associated with the development of thyroid cancer and tumors or hyperplasia in other endocrine organs. It is caused by mutations in the RET gene and can be phenotypically classified into MEN types 2A and 2B. MEN2B is often sporadic resulting from a spontaneous mutation, M981T. A positive paternal germline selection has been reported for this mutation.
\end{abstract}

Methods: We analyzed the V804M mutation in the RET gene which also affects the intracellular domain of the protein but results in a different phenotype, MEN2A. We compared the observed and expected frequencies of the V804M mutation and the paternal and maternal germline transmission frequency of V804M mutation in three previously reported multigenerational families.

Results: Our analysis indicates that the observed frequency of the V804M mutation is significantly greater than the expected frequency suggesting positive germline selection $(\mathrm{P}<0.001)$. Furthermore, comparative analysis of observed versus expected transmission frequencies from affected parents shows a higher maternal germline transmission frequency $(\mathrm{P}=0.001)$.

Conclusions: Our results suggest that in the RET gene, positive germline selection may extend to mutations other than M918T and, furthermore, at least for the V804M mutation in these families, there is evidence for maternal germline selection.

Keywords: RET gene; Germline selection; V804M mutation; Multiple endocrine neoplasia type 2

Manuscript accepted for publication December 01, 2016

${ }^{a}$ The Neuro-genetics Institute, 501 Elmwood Avenue, Sharon Hill, PA, USA bJersey Shore University Medical Center, Meridian Health, 1945 State Route 33, Neptune, NJ, USA

${ }^{c}$ Neuroscience Institute, Saint Francis Medical Center, 601 Hamilton Avenue, Trenton, NJ, USA

${ }^{\mathrm{d} C}$ Corresponding Author: Raji P. Grewal, Seton Hall University, Neuroscience Institute, Saint Francis Medical Center, 601 Hamilton Ave., Trenton, NJ, USA. Email:RGrewal@stfrancismedical.org

doi: https://doi.org/10.14740/wjon970e

\section{Introduction}

There is increasing interest in the biological phenomenon of germline selection of mutations and this has been the focus of studies of certain diseases including achondroplasia and Apert syndrome. More recently, these studies have expanded to include an investigation of multiple endocrine neoplasia type 2 (MEN2) [1]. MEN2 is an autosomal dominant cancer syndrome associated with the development of tumors in multiple endocrine organs, the most characteristic being the occurrence of highly penetrant medullary thyroid cancer. It is caused by germline mutations of RET proto-oncogene (REarranged during Transfection) that encodes a transmembrane receptor which has tyrosine kinase activity and functions in the development of sympathetic, parasympathetic and enteric neurons [2]. MEN2 can be sub-typed based on genotype/phenotype correlation into MEN2A, familial medullary thyroid carcinoma (FMTC), and MEN2B. MEN2A is more common, accounting for $70-80 \%$ of all cases of MEN2 and is characterized by the presence of thyroid carcinoma, unilateral or bilateral pheochromocytoma and parathyroid adenoma/hyperplasia. FMTC, which accounts for about $10-20 \%$ of all cases of MEN2, is phenotypically characterized by the presence of only medullary thyroid carcinoma. Mutations in the exons encoding the intracellular domain of the RET gene are common and mutations within exons 13 and 14 have been associated with MEN2A and FMTC, while mutations in exon 16 cause MEN2B [3].

MEN2B is less common, accounting for $5 \%$ of all cases of MEN2 and is characterized by presence of medullary thyroid cancer, pheochromocytoma, a marfinoid habitus and ganglioneuromas of the intestinal tract mucosa. It is often sporadic with almost $50 \%$ of index cases resulting from a spontaneous germline mutation. Interestingly, 95\% of all sporadic MEN2B mutations involve c.2943T $>C$ resulting in M981T substitution affecting the intracellular tyrosine kinase portion of the protein. Based upon direct analysis of this specific point mutation in human sperm samples, it was noted that its frequency is much higher than that anticipated based upon several models of expected results. It was concluded that there is positive selection of this mutation in male germline cells [1].

Feldman et al [4] reported the substitution of the valine with methionine at codon 804 (V804M) in exon 14 of the RET gene in two families with medullary thyroid carcinoma (MTC). 
Family 1

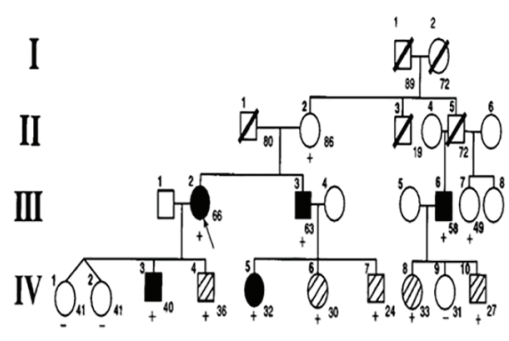

Family 2

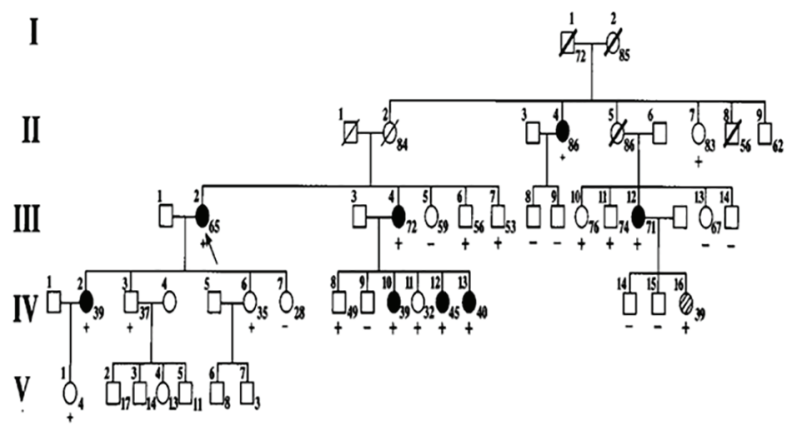

Figure 1. Pedigrees of families 1 and 2 (reprinted from [4]).

The same mutation was reported in a large kindred diagnosed with MEN2A by Shifrin et al [3]. Members who tested positive for the V804M mutation as in these families developed MTC, C-cell hyperplasia $(\mathrm{CCH})$, papillary thyroid carcinoma (PTC) or primary hyperparathyroidism (PHPT). MEN2A is transmitted in autosomal dominant fashion; however, there seemed to be disproportionately higher number of V804M carriers in these families. We report our analysis of the unusual transmission pattern of the V804M mutation in the combined clinical and genotype data in these three pedigrees.

\section{Methods}

All of the data used for this study were obtained from published studies. Family 1, a four-generation family with 26 members and family 2, consisting of 48 individuals belonging to a fivegeneration family with multiple members affected with MTC, were both reported by Feldman et al (Fig. 1) [4]. The third family consisting of 107 members belonging to a three-generation Italian-American family was previously described by Shifrin et al (Fig. 2) [3]. The total number of individuals from V804M positive parents in each generation was identified for all three families and the observed and expected numbers of individuals carrying this mutation were tabulated. The number of individuals with paternal and maternal inheritance was determined for each generation and the observed and expected V804M germline transmission frequencies were also tabulated. The final expected and observed data for the V804M muta- tion for each family and all families combined are summarized along with our analysis (Table 1). A Chi-square goodness-offit test was used to compare observed frequencies to expected frequencies using SPSS version 16.0 (SPSS Inc., Chicago, IL) statistical software.

\section{Results}

As expected, the V804M mutation in the RET proto-oncogene is transmitted in an autosomal dominant fashion. When the mode of inheritance is autosomal dominant, as observed in these families, the frequency of the affected progeny of an affected individual is expected to be $50 \%$.

The observed frequency of V804M carriers in family 3 (40 positive of 53 subjects) is significantly higher than the expected frequency of $50 \%$ (26.5 of 53 subjects) $(\mathrm{P}<0.001)$. Similarly, the observed frequency for the V804M carriers in family 1 (13 positive of 15 subjects) and family 2 (20 positive of 29 subjects) is higher than the expected frequency of $50 \%$. However, due to the small sample size, statistical analysis was not performed for families 1 and 2 . The total observed frequency for all three families combined was significantly higher $(\mathrm{P}<$ 0.001 ) than the expected frequency of $50 \%$ (Table 1).

We also analyzed the possible parental effect on the frequency of transmission of the V804M mutation. Assuming no parental influence, there should be no difference in frequency for whether a child carrying the V804M mutation inherited this mutation through maternal or paternal transmission. In fami-

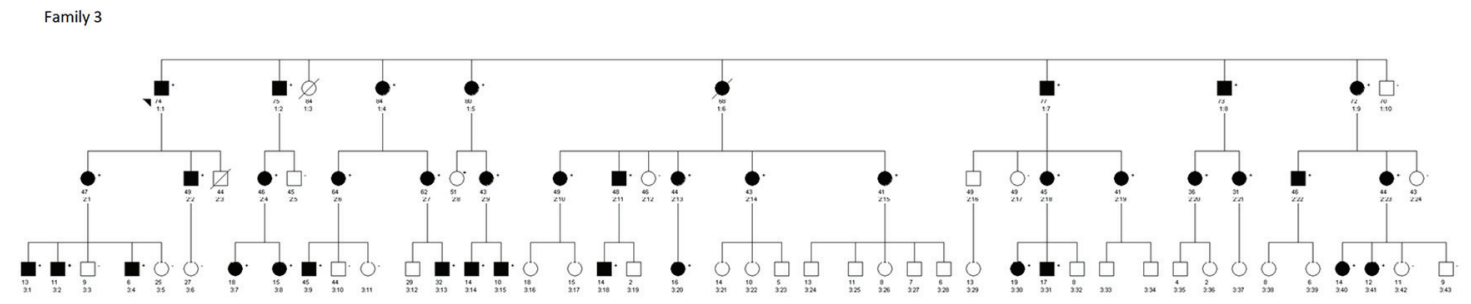

Figure 2. Pedigree of 107 members of a multigenerational Italian-American family with autosomal dominant transmission of the V804M RET mutation causing MEN2A in the affected individuals. Solid circles and squares indicate individuals positive for the V804M RET mutation. 


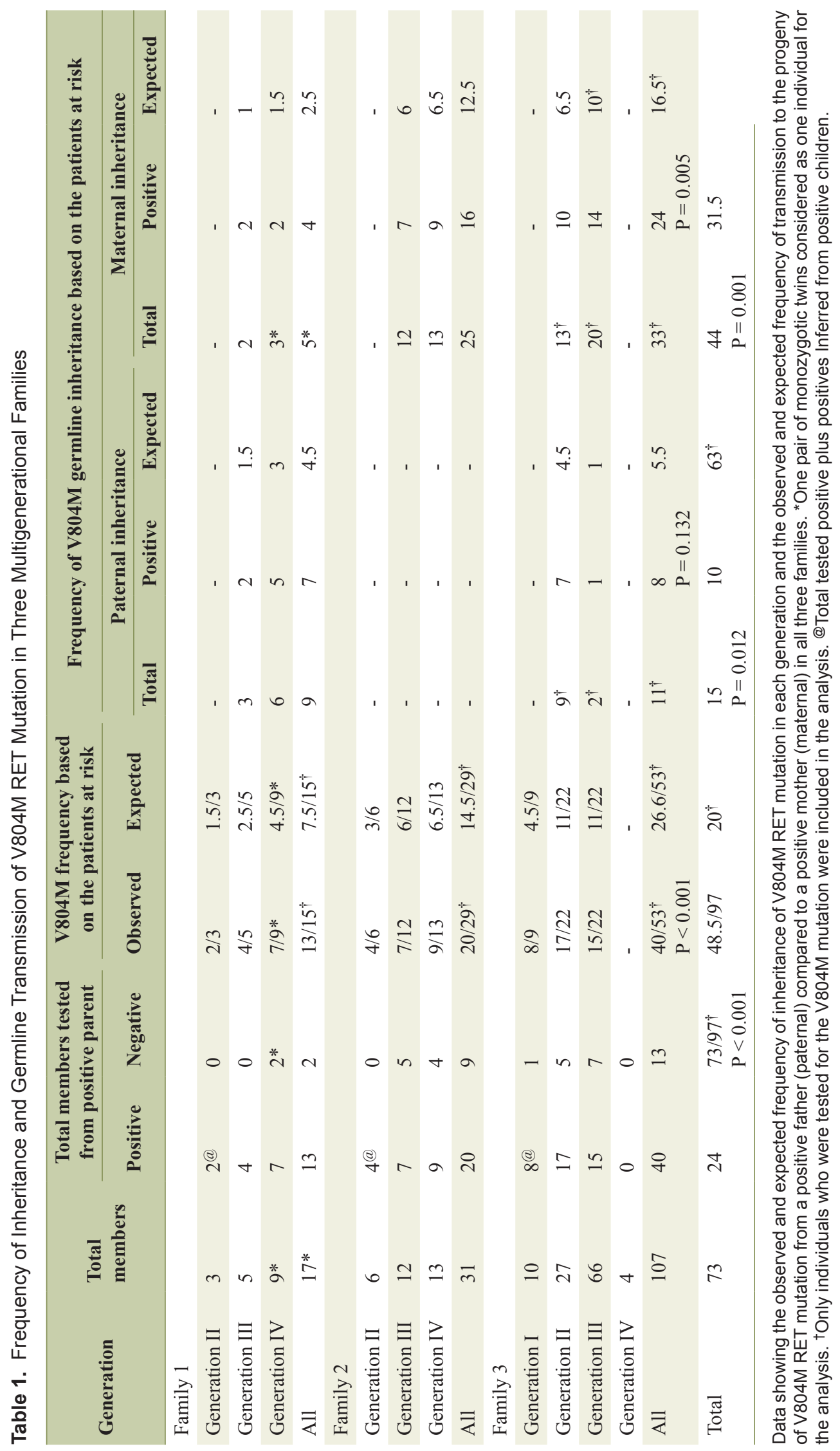


lies 1 and 3, we observed preferential selection for the mutant allele irrespective of whether it was maternal or paternal inheritance. In family 2 , the maternal transmission showed germline selection for the mutant allele; however, in this family, data for the offspring of affected males were not available for analysis. Again, due to the small sample size, the statistical analysis was not performed comparing paternal and maternal inheritance frequencies for families 1 and 2 individually. In family 3, the maternal transmission rate ( 24 positive of 33 subjects) was significantly higher $(\mathrm{P}=0.005)$ than the expected. Although the paternal transmission frequency (eight positive of 11 subjects) was higher than the expected (5.5 of 11), it was not statistically significant $(\mathrm{P}=0.132)$. The observed frequency of combined paternal transmission from the three families (15 of 20 subjects) was significantly higher than the expected frequency of $50 \%$ (10 of 20 subjects) $(\mathrm{P}=0.012)$. Similarly, the observed frequency of the pooled maternal transmissions in the three families (44 of 63 subjects) was significantly higher than the expected frequency of $50 \%$ (31.5 of 63 subjects) $(\mathrm{P}=0.001)$ (Table 1).

\section{Discussion}

Our analysis of these three families shows a significant deviation from the expected $50 \%$ ratio of an autosomal dominant trait suggesting positive germline selection for the V804M mutant allele (Table 1). These results support the conclusion of prior research studying positive germline selection in the RET gene and indicate this phenomenon may occur with mutations other than M918T. Our results support selection of the V804M mutation in all germ cells not only in paternal gametes (Table 1).

Previous studies have proposed biological mechanisms of how point mutations could confer a selective advantage in male germ cells. The process of oogenesis starts during development in utero as germ cells differentiate into oogonia that ultimately disappear by birth. Unlike males, at birth, females possess all of the oocytes they will ever have. At 5 months of gestation, there is a peak of about 7 million germ cells which then falls to $1-2$ million at birth. Throughout the reproductive lifespan of women, there is a loss of oocytes predominantly by atresia [5]. In theory, positive germline selection of those gametes carrying the V804M RET mutation could occur any time after oogenesis starts and could continue throughout the reproductive life. Based upon studies of mouse spermatogonial cells, it has been proposed that positive selection in sperm cells involves modification of the signal transduced from the mutated RET gene [6]. Although the role of this gene in oogenesis has not been investigated as extensively as with spermatogenesis, there is a report of the induction of maturation of xenopus oocytes with activated RET products [7]. This study provides a biological basis of a potential role of this gene in oocyte differentiation that could result in positive selection in maternal germline.

Our study of these three families suggests that positive germline selection occurs in both paternal and maternal gametes carrying the V804M mutation. At the present time, ova are not as amenable to the kinds of studies that are possible with spermatozoa to confirm and extend our results regarding maternal germline selection at a cellular level. Although the concept of selection occurring at the level of gametes was first proposed by Hastings, only recently has it been possible to investigate and provide experimental support for this phenomenon [8-10]. These investigations are important as they have implications for many broad fields ranging from clinical genetics to evolutionary biology.

\section{Acknowledgments}

We are grateful for the support of the Neurogenetics Foundation. We thank John Urschel MD for his assistance with statistical analysis.

\section{Conflicts of Interest}

The authors declare that there are no conflicts of interest regarding the publication of this paper.

\section{References}

1. Choi SK, Yoon SR, Calabrese P, Arnheim N. Positive selection for new disease mutations in the human germline: evidence from the heritable cancer syndrome multiple endocrine neoplasia type 2B. PLoS Genet. 2012;8(2):e1002420.

2. Carlson KM, Bracamontes J, Jackson CE, Clark R, Lacroix A, Wells SA, Jr., Goodfellow PJ. Parent-of-origin effects in multiple endocrine neoplasia type 2B. Am J Hum Genet. 1994;55(6):1076-1082.

3. Shifrin AL, Xenachis C, Fay A, Matulewicz TJ, Kuo YH, Vernick JJ. One hundred and seven family members with the rearranged during transfection V804M proto-oncogene mutation presenting with simultaneous medullary and papillary thyroid carcinomas, rare primary hyperparathyroidism, and no pheochromocytomas: is this a new syndrome--MEN 2C? Surgery. 2009;146(6):998-1005.

4. Feldman GL, Edmonds MW, Ainsworth PJ, Schuffenecker I, Lenoir GM, Saxe AW, Talpos GB, et al. Variable expressivity of familial medullary thyroid carcinoma (FMTC) due to a RET V804M (GTG-->ATG) mutation. Surgery. 2000;128(1):93-98.

5. Faddy MJ, Gosden RG. A model conforming the decline in follicle numbers to the age of menopause in women. Hum Reprod. 1996;11(7):1484-1486.

6. Miles DC, van den Bergen JA, Wakeling SI, Anderson RB, Sinclair AH, Western PS. The proto-oncogene Ret is required for male foetal germ cell survival. Dev Biol. 2012;365(1):101-109.

7. Grieco D, Santoro M, Dathan NA, Fusco A. Activated RET oncogene products induce maturation of xenopus oocytes. Oncogene. 1995;11(1):113-117.

8. Hastings IM. Potential germline competition in animals and its evolutionary implications. Genetics. 
1989;123(1):191-197.

9. Hastings IM. Germline selection: population genetic aspects of the sexual/asexual life cycle. Genetics. 1991;129(4):1167-1176.
10. Goriely A, McVean GA, Rojmyr M, Ingemarsson B, Wilkie AO. Evidence for selective advantage of pathogenic FGFR2 mutations in the male germ line. Science. 2003;301(5633):643-646. 